\title{
La gestion du risque infectieux en implantologie
}

\section{Management of infection risk in implantology}

MOTS-CLEFS :
- Contamination,
bio-nettoyage,
stérilisation,
ergonomie
KEYWORDS:
- Contamination,
organic cleaning,
sterilization,
ergonomics

AOS 2014;269:28-33 DOI: $10.1051 /$ aos/2014306 (c) EDP Sciences 2014

\section{Résumé}

La gestion du risque infectieux constitue un garant indispensable pour la réussite de toute implantation. Le praticien doit s'assurer de respecter avec rigueur tous les principes d'asepsie et d'ergonomie en pré-, per-, et post-opératoire. Ces principes concernent essentiellement la chaine de stérilisation pour tous les instruments et les outils réutilisables, la préparation et l'organisation de la salle d'intervention, la préparation du patient et de l'équipe opératoire, l'ergonomie et la gestuelle maîtrisée en peropératoire, et enfin l'évacuation des déchets et l'entretien des locaux.

\author{
Abstract
}

Management of infection risk is a guarantee of success for any implementation. The practitioner must ensure compliance with all rigor principles of asepsis and ergonomics at pre, per and post operative. These principles concern mainly the chain of sterilization for all instruments and reusable tools, preparation and organization of the operating room, preparing patients and surgical team, ergonomics and movement control in peroperation, and finally waste disposal and local maintenance.

Mohssine BAGUI, Chirurgien dentiste, spécialiste en prothèse adjointe.
Leila FAJRI, Professeur assistant en prothèse adjointe.
Bouabid EL MOHTARIM, Professeur de l'enseignement supérieur en prothèse
adjointe. Chef de service d'odontologie à l'hôpital d'instruction militaire Mohammed V
à Rabat.
$\begin{aligned} & \text { Nadia MERZOUK, Professeur de l'enseignement supérieur en prothèse adjointe. } \\ & \text { Chef de service de prothèse adjointe CCTD Rabat. }\end{aligned}$


$\mathrm{L}^{2}$ a gestion du risque infectieux constitue une obligation dans la pratique de toutes les disciplines de santé. Cela consiste en la maîtrise des mesures d'asepsie afin d'empêcher tout apport exogène de micro-organismes.

En implantologie, discipline à haut risque infectieux, la réussite de l'acte chirurgical n'est confirmée qu'après ostéo-intégration de l'implant. Ce phénomène est lié principalement à la qualité osseuse, au type d'implant, aux techniques et aux méthodes chirurgicales mais aussi aux conditions de travail. L'ergonomie et la maîtrise de la gestuelle et de l'instrumentation complexe et multiples, ainsi que le respect rigoureux de la chaîne d'asepsie constituent les éléments clés assurant l'ostéo-intégration de l'implant $[1,2,3]$.

La prévention de l'infection en implantologie est essentiellement une lutte contre la contamination, qui peut être soit directe, soit indirecte :

I la contamination indirecte : cette contamination est essentiellement aérienne, liée à la présence des spores et des micro-organismes dans l'air de la salle d'intervention, du fait de la présence humaine ainsi que de l'utilisation du matériel rotatif [3] ;

I la contamination directe : cette contamination est due aux micro-organismes du patient (flore cutanée respiratoire ou orale), aux erreurs de stérilisation, ou bien au comportement inadéquat du praticien. Afin d'assurer la réalisation du geste implantaire avec un minimum de risque infectieux possible, le praticien doit travailler dans des conditions d'hygiène et d'asepsie répondant aux règles de la bonne pratique et aux précautions standards pour un acte invasif avec projection du liquide biologique. Un ensemble de règles pré-, per- et post-opératoires doit être strictement respecté pour atteindre ce but [3].

\section{PRÉPARATION DU MATÉRIEL}

Avant de débuter tout acte implantaire, le praticien doit contrôler la disponibilité du matériel nécessaire ou probablement nécessaire pour l'implantation, tout en préparant un stock de rechange en cas de besoin. De plus, il faut qu'il s'assure de l'état stérile du matériel réutilisable $[1,4,5]$.

\section{Chaîne de stérilisation}

La stérilisation des dispositifs médicaux réutilisable exige le respect d'une suite d'étapes bien définies permettant d'assurer l'état stérile.

\section{Décontamination ou pré-désinfection}

Elle consiste à tremper l'instrument dans un bac de détergent désinfectant afin de faciliter le nettoyage et de minimiser la charge bactérienne pour réduire le risque de contamination lors de l'étape suivante (fig. 1).

\section{Nettoyage}

Manuel, automatique ou ultrasonique, le nettoyage est effectué en utilisant une solution détergente désinfectante pour éliminer toute salissure et tous débris organiques (fig. 2).

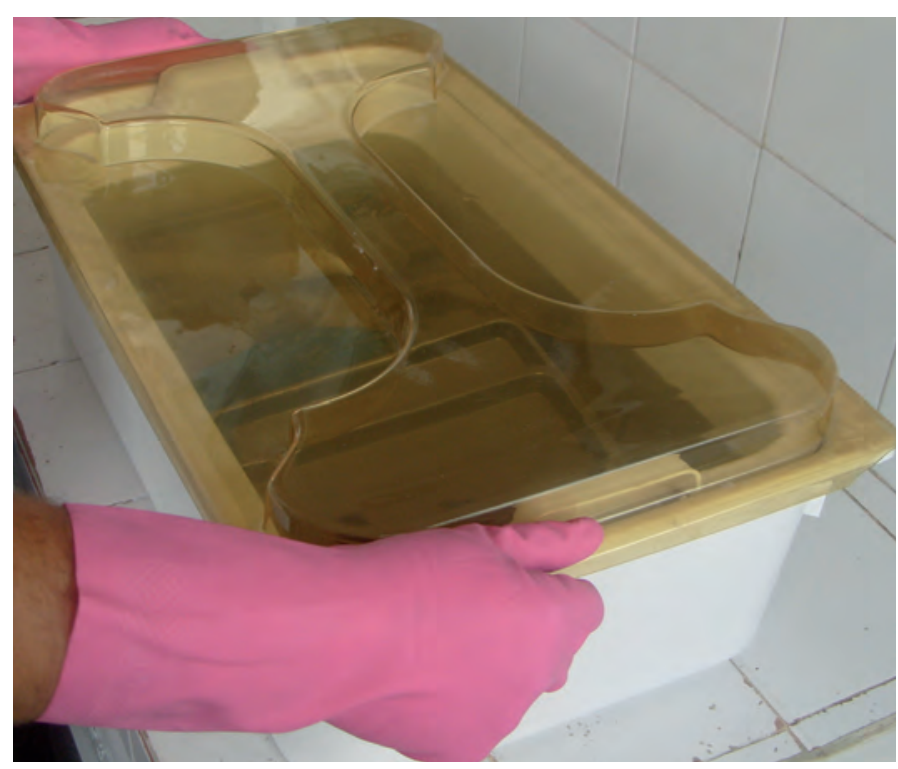

$\triangle$ Fig. 1 :

Les instruments sont baignés dans un bac contenant un décontaminant pour minimiser le risque de contamination lors du nettoyage.

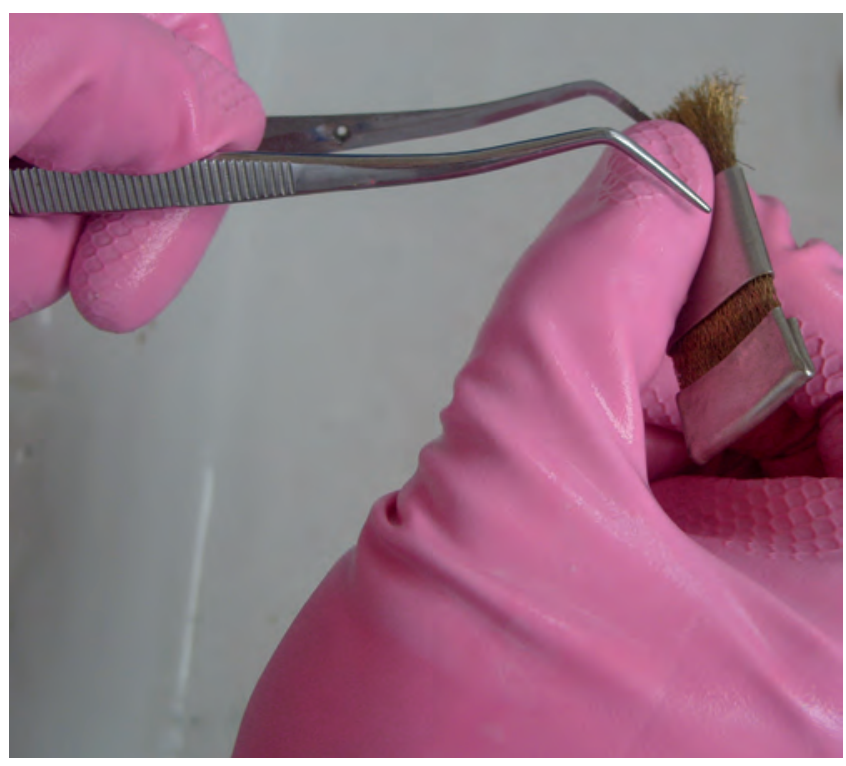

$\triangle$ Fig. 2 :

Le nettoyage mécanique manuel des instruments. 


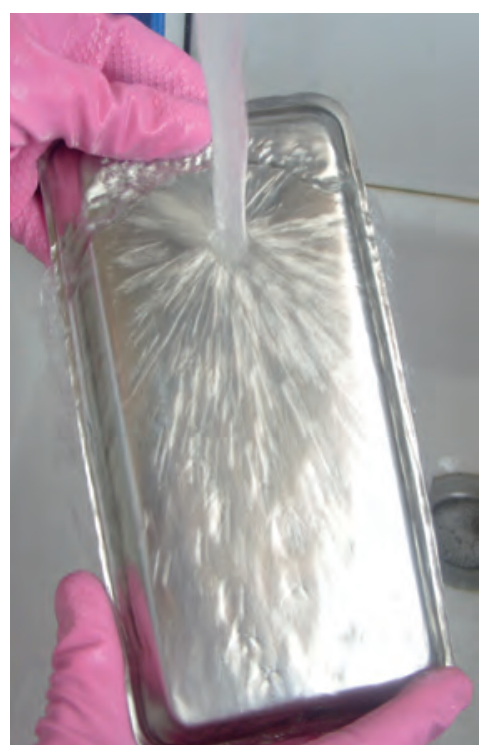

$\triangle$ Fig. 3 :

Le rinçage abondant sous l'eau coulante.

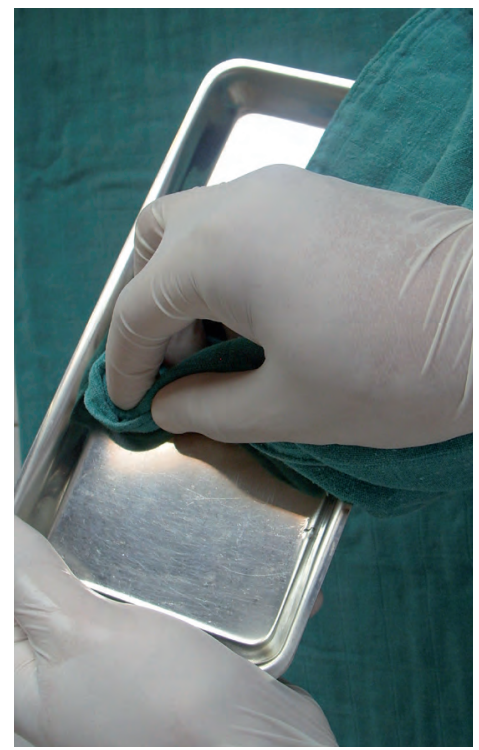

$\triangle$ Fig. 4 :

Le séchage avec un champ en tissu stérile.

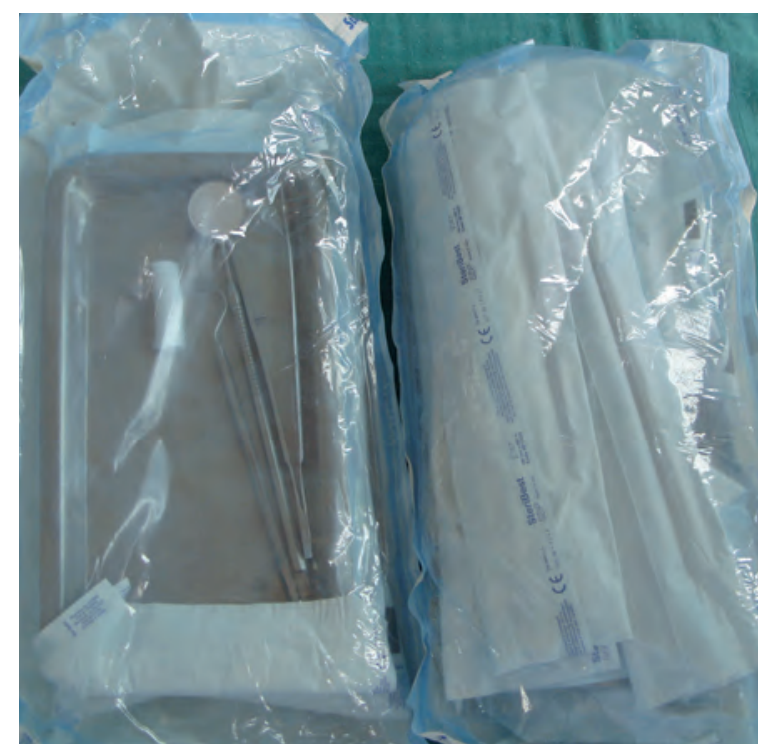

$\triangle$ Fig. 5 :

Le conditionnement dans un sachet afin de conserver les instruments à l'état stérile.

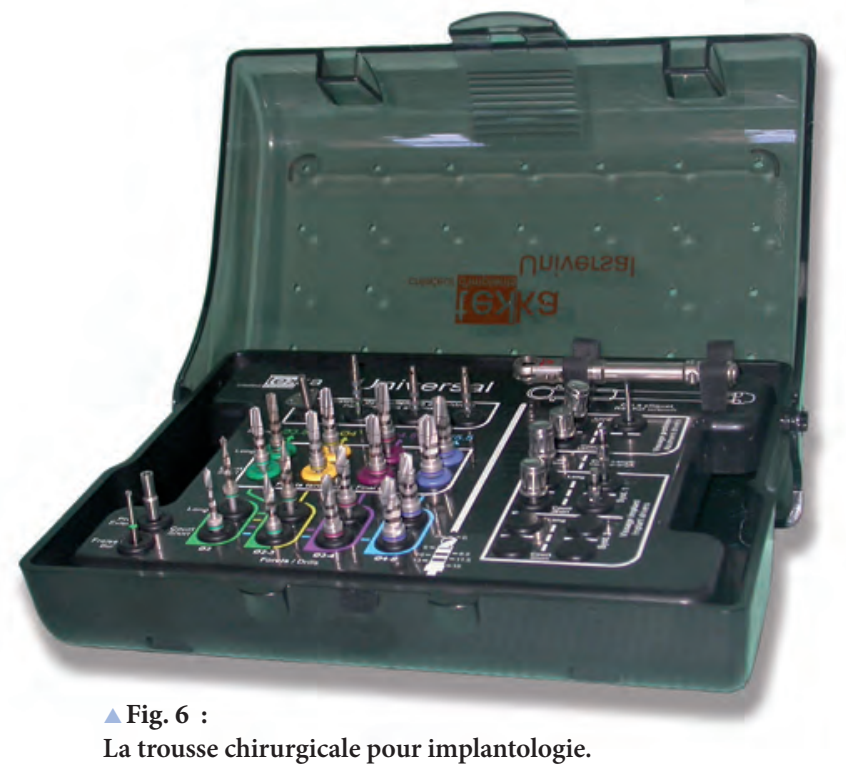

Rinçage et séchage

Il faut éliminer toute trace de solution par un rinçage abondant, suivi d'un séchage excluant toute trace d'humidité (fig. 3, 4).

\section{Conditionnement et stérilisation}

Le conditionnement permet le stockage des instruments à l'état stérile en préservant le résultat obtenu après l'étape de stérilisation (fig. 5).

La stérilisation à la vapeur d'eau est le procédé de référence à l'état actuel, en utilisant une pression de 2 bars, une température de $134^{\circ}$, durant 18 minutes. Le contrôle du bon déroulement du cycle est assuré par l'enregistrement de ce dernier, l'intégrateur phy- sico-chimique et le test de Bowie-Dick participent à la vérification et la traçabilité des instruments.

\section{Désinfection}

Concernant les instruments thermosensibles qui sont très rares en implantologie, une désinfection est indiscutable avant chaque utilisation. L'instrument est alors trempé dans une solution normée désinfectante durant 2 heures, juste avant l'utilisation. Le matériel désinfecté ne peut être stocké. C'est un état momentané.

Lorsqu'une intervention de chirurgie implantaire est programmée, il faut préparer l'ensemble du matériel spécifique à cette chirurgie, en contrôlant la disponibilité des implants, vis de couverture, pilier de cicatrisation, trousse spécifique de la chirurgie implantaire, sans oublier le stock d'instruments stériles à usage uniques. En cas de manque, le matériel est commandé en respectant le délai de livraison.

Le stock d'implant : une semaine avant l'intervention, le praticien doit s'assurer des implants disponibles selon le système implantaire choisi, la longueur, le diamètre et l'état de surface, ainsi que des vis de couverture et de cicatrisation.

La trousse chirurgicale rassemble l'ensemble de l'instrumentation nécessaire pour la préparation et la mise en forme du site à implanter. Dans chaque trousse, on doit avoir une cassette autoclavable, des forets spécifiques à chaque système implantaire, les prolongateurs des forets, les tiges de parallélisme, les tournevis, les connecteurs d'implants ou drivers. L'ensemble de la trousse doit subir les différentes étapes de la chaîne de stérilisation à l'autoclave [4] (fig. 6). 
L'instrumentation rotative doit subir un traitement externe ainsi qu'un traitement interne avant le conditionnement et la stérilisation. Le traitement externe consiste en une décontamination, un nettoyage par brossage doux, suivi d'un séchage. Le traitement interne peut être manuel suivant les instructions du fabriquant ou mécanique par des systèmes mécanisés qui réalisent le nettoyage et la lubrification de l'instrument [6].

\section{SALLE D'INTERVENTION : PRÉPARATION ET ORGANISATION}

La préparation et l'organisation de la salle d'intervention ont pour objectifs d'assurer un environnement de travail respectueux d'une hygiène rigoureuse et d'organiser un plateau technique pour avoir une ergonomie de travail respectant la chaîne d'asepsie.

Pour atteindre ces objectifs, la salle d'intervention doit répondre à plusieurs critères :

Ia bonne aération : l'air doit être constamment renouvelé pour diminuer sa concentration potentielle en micro-organismes ;

Ile revêtement du sol, des murs et du plafond doit être non poreux et facile à nettoyer ;

$\checkmark$ la salle ne doit pas être encombrée, sans objets décoratifs, ni plantes vertes ni meubles roulants ;

Ia disponibilité d'une eau filtrée pour le lavage chirurgical des mains, d'une eau technique pour les différents appareils, et d'une eau potable pour une autre utilisation.

La préparation de la salle débute par le nettoyage et la désinfection, suivis de l'installation du plateau technique avec la mise en place des tables et le matériel indispensable à l'intervention chirurgicale $[1,4,6]$.

\section{Bionettoyage des locaux}

Les locaux comportent trois types de zones en fonction $\mathrm{du}$ risque potentiel de contamination. Chaque zone nécessite un traitement différent par rapport aux autres zones. On distingue la zone 1 dite administrative, la zone 2 dite potentiellement contaminée, et la zone 3 nommée protégée :

$\checkmark$ la zone 1 : nettoyage quotidien dit domestique;

$\checkmark$ la zone 2 : bionettoyage quotidien avec un produit détergent désinfectant normé ;

$\checkmark$ la zone 3 : bionettoyage après toute intervention avec un produit détergent désinfectant normé.

Ce bionettoyage doit être réalisé en allant de la zone la plus propre vers la zone la plus sale selon la technique du double seau. Il se fait en trois étapes successives :

\évacuation des déchets de soins ; nettoyage associant une action mécanique et une action chimique ;

$\checkmark$ application d'un désinfectant $[1,4,7]$.

\section{Préparation du plateau technique}

On commence par désinfecter les plans de travail qui sont nettoyés par des lingettes pré- imprégnées. Puis on installe le champ stérile sur la table chirurgicale préalablement désinfectée, et ceci en ouvrant l'emballage au-dessus de la table puis on le laisse tomber dessus. Seule la partie horizontale du champ est considérée stérile. Ensuite on passe à l'installation du plateau chirurgical qui comporte toute l'instrumentation commune à toute chirurgie buccale ainsi que la trousse chirurgicale spécifique au système implantaire choisi (fig. 6). À la fin, on installe la table du moteur chirurgical désinfecté ainsi que son irrigation stérile. Le moteur doit être installé sur un plan de travail préalablement désinfecté et couvert par un champ stérile, et par un emballage transparent stérile pour manipuler les boutons sans risque de contamination.

Sur le moteur, on connecte les tubulures de l'irrigation stérile qui est constituée de sérum physiologique disposé en poche nylon transparente. Ces tubulures sont stériles et sous sachets (fig. 7).

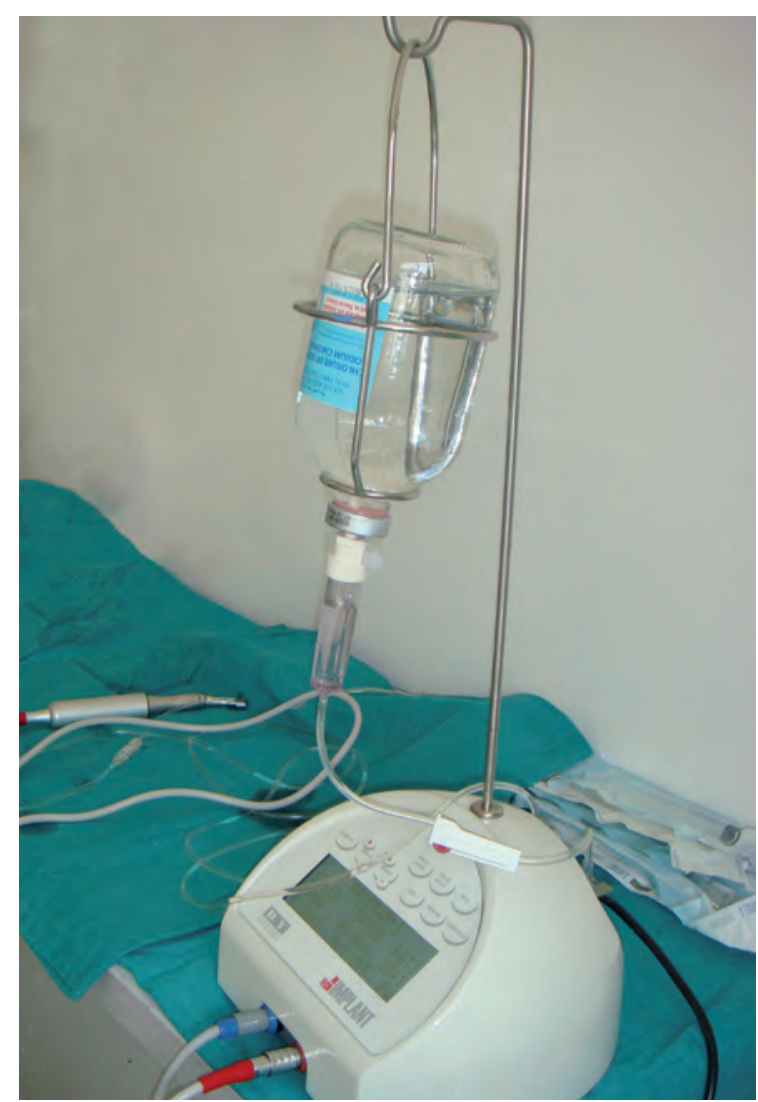

$\triangle$ Fig. 7 :

Le moteur installé sur un champ stérile et muni d'un système d'irrigation au sérum physiologique. 
Le bras du scialytique est toujours couvert par un champ en tissu stérile afin d'éviter toute contamination lors de sa manipulation.

Il faut noter que cette installation est toujours réalisée avec une tenue adaptée $[1,4]$.

\section{PRÉPARATION ET MISE EN PLACE DES DIFFÉRENTS ACTEURS}

\section{Préparation du patient}

Avant l'intervention, le patient doit être préparé selon un protocole bien clair répondant aux règles générales d'asepsie :

$\checkmark$ l'assainissement de la cavité buccale et élimination de tous les foyers infectieux ;

$\checkmark$ la prémédication du patient, anxiolytique, antibiotique, anti-inflammatoire, corticoïde, antalgique, antihémorragique ;

I la désinfection buccale avec un bain de bouche à base d'iode ;

l'habillage du patient : casaque, sur-chaussures, charlotte ;

l'installation du patient sur fauteuil sans rien toucher;

$\checkmark$ la désinfection cutanée péribuccale à l'aide d'une compresse imbibée de bétadine ou de chlorhexidine, suivie d'un badigeonnage endobuccal (fig. 8) ;

$\checkmark$ le drapage du patient avec des champs de type chirurgical stérile (recouvrement stérile) $[1,3,4,6]$.

\section{Préparation de l'équipe opératoire}

Des règles rigoureuses concernant l'hygiène et la tenue du personnel visent à éviter le risque de contamination de patient à praticien, ou de praticien à patient, ou encore de patient à patient. Pour réussir l'implantation, la préparation de l'équipe opératoire doit répondre au protocole suivant :

Ile port d'une tenue spécifique non stérile mais propre ;

$\checkmark$ le port en ordre d'une charlotte, d'un masque, des lunettes, d'un sabot, et des sur-chaussures ;

$\checkmark$ le lavage chirurgical des mains;

$\checkmark$ le port d'une tenue stérile (blouse chirurgicale ou casaque stérile à usage unique) et des gants chirurgicaux stériles $[1,3,4,6]$.

\section{Lavage chirurgical}

Le lavage chirurgical est réalisé avant tout geste chirurgical invasif majeur dans le but de réduire au maximum la flore commensale et les squames cutanés des mains et des avant-bras, ainsi que d'éliminer la flore transitoire. Ce lavage s'effectue en trois temps :
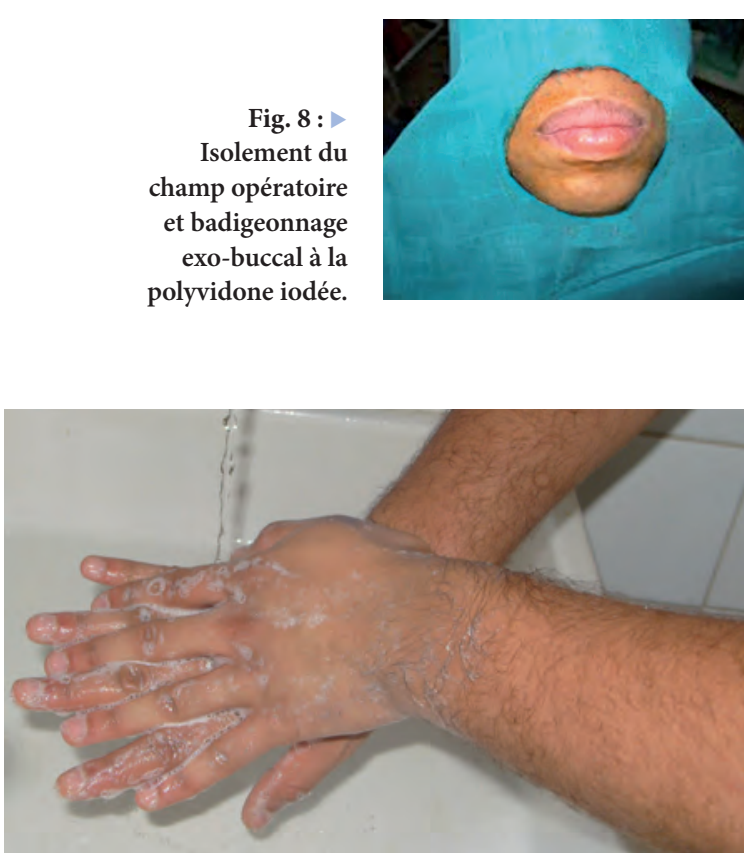

$\triangle$ Fig. 9 :

Lavage des mains en utilisant un produit désinfectant.

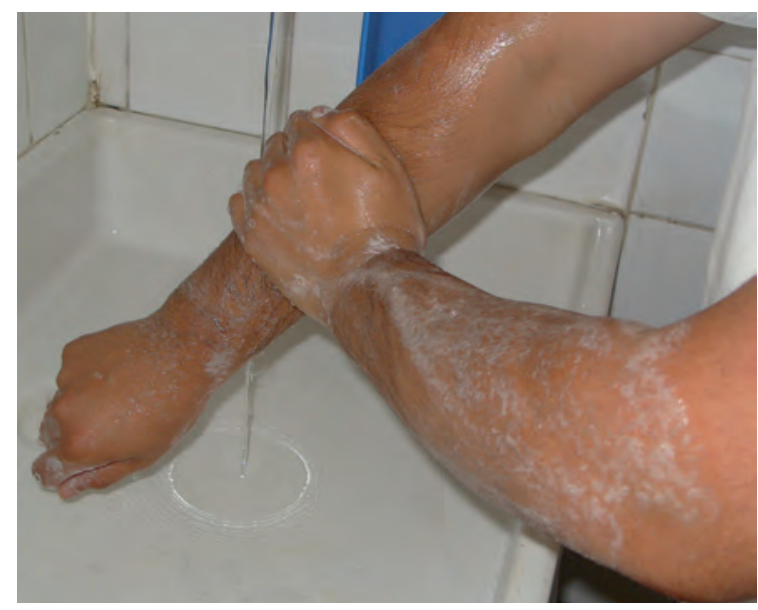

Fig. 10 :

Lavage des avant-bras.

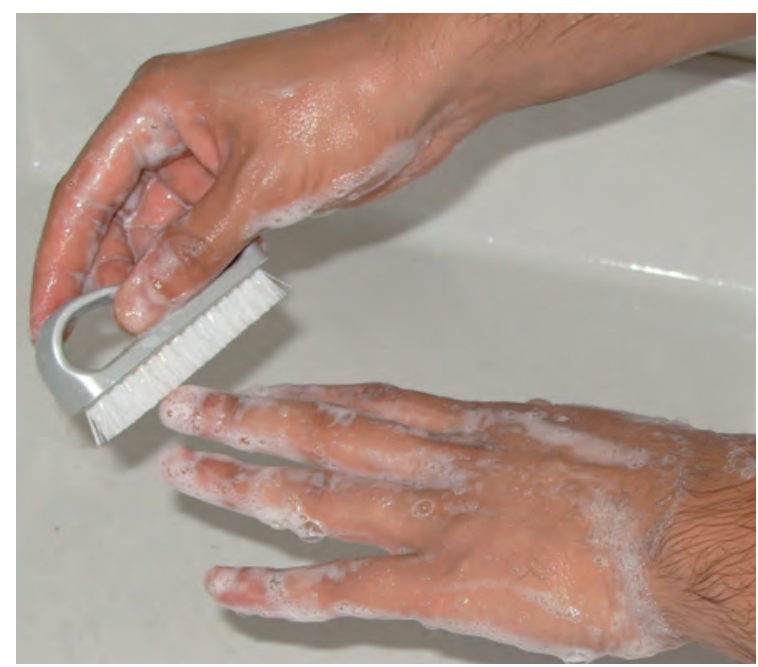

$\triangle$ Fig. 11 :

Lavage des angles et des parties interdigitales. 


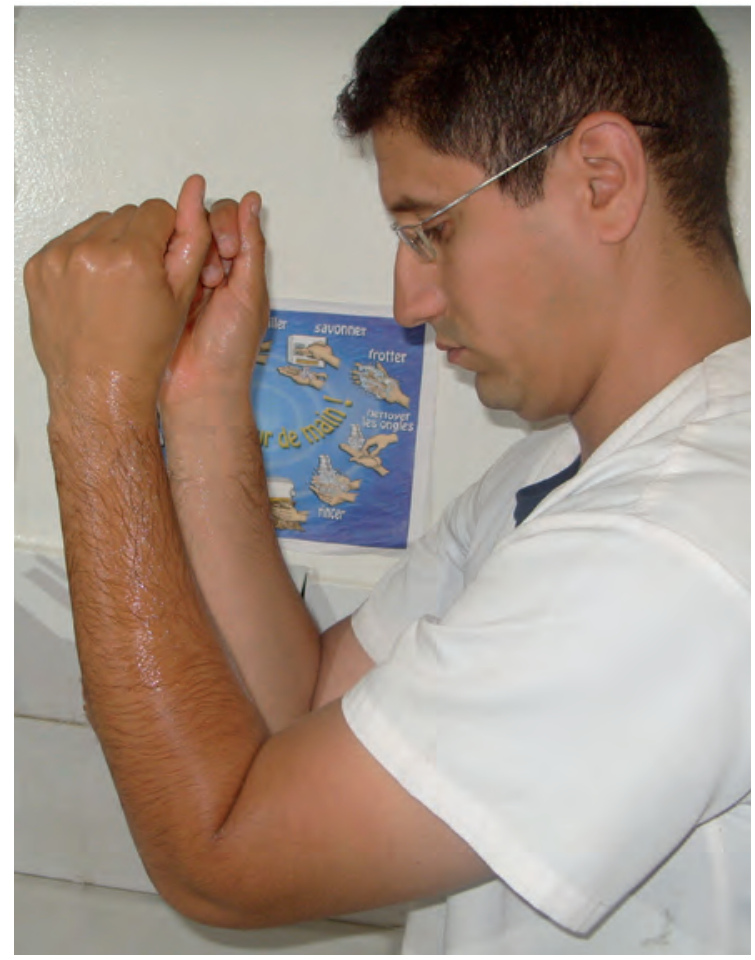

Fig. 12 :

Rinçage final en gardant toujours les coudes vers le bas.

$\checkmark$ laisser l'eau couler sur les mains et les avant-bras pendant 30 secondes, suivi d'un mouillage et lavage en utilisant une solution antiseptique à large spectre avec rinçage (fig. 9, 10) ;

\brossage des ongles uniquement avec rinçage, à l'aide d'une brosse à usage unique comprenant un côté éponge en mousse imbibée ou non de savon liquide, et un côté brosse avec curette à ongle (fig. 11) ;

I un dernier lavage et rinçage des mains et des manchettes, suivi d'un rinçage final en gardant les coudes toujours vers le bas (fig. 12).

Le temps nécessaire, en ajoutant le temps du rinçage, est de 5 à 8 minutes en accord avec le protocole national des comités de lutte contre les infections nosocomiales. Le séchage est très important car l'humidité favorise la pullulation microbienne et ne permet pas d'assurer la stérilisation d'une casaque chirurgicale surtout si celle-ci est en tissu. Dans ce but, on utilise un ou deux essuie-mains stériles des extrémités vers les coudes $[1,3,4,6]$.

\section{Habillage}

Une fois le lavage des mains terminé, l'assistante tient le pack contenant la tenue et les serviettes stériles et le présente ouvert au praticien qui va se servir des serviettes pour se sécher les mains, et enfiler la tenue tout en écartant les mains. Il faut toujours insister sur le fait que l'extérieur de la tenue ne doit pas être touché. Ensuite, les cordons sont noués, soit par l'assistante, soit par le praticien.

Pour enfiler les gants, l'assistante doit ouvrir l'emballage sans toucher l'intérieur. Le praticien récupère les gants stériles dans leur sachet papier et les enfile en ne touchant avec les doigts que la partie interne des gants. L'assistante va se laver les mains, enfiler la blouse et les gants dont elle aura auparavant ouvert les emballages plastiques $[1,3,4,6]$.

\section{Ergonomie et gestuelle en peropératoire}

La gestuelle bien raisonnée et méthodique permet une parfaite maîtrise de l'asepsie, et constitue la clé de réussite de l'intervention chirurgicale.

L'ergonomie rigoureuse et parfaite impose une organisation réfléchie et fonctionnelle lors de toutes les étapes de l'intervention $[4,6]$.

I La salle d'intervention est préparée avant l'arrivée $\mathrm{du}$ patient, alors que les instruments doivent être installés à l'arrivée de ce dernier afin de minimiser le temps au cours duquel les dispositifs sont hors conditionnement même couverts d'un champ stérile. I L'instrumentation est placée par le praticien avec soin selon l'ordre opératoire. Les champs et emballages stériles ne sont enlevés que juste avant l'intervention afin d'éviter tout contact septique.

I Lors de l'acte chirurgical, l'assistante doit assurer un dégagement du site opératoire permettant une vue simple pour le praticien avec une aspiration chirurgicale précise et efficace.

I La mise en place de l'implant nécessite une attention particulière. Lors de l'ouverture de l'emballage et la pose d'implant, la surface de ce dernier ne doit toucher aucune surface. Le premier contact de l'implant doit être avec l'os et le sang en évitant toute interférence.

I Après sutures, quelques compresses imbibées de sérum physiologique permettent de comprimer légèrement le site opératoire $[4,6]$.

\section{Procédures postopératoires}

Après le départ du patient, en lui expliquant son ordonnance, l'équipe opératoire doit réaliser un nettoyage de la salle d'intervention, ainsi qu'un traitement biomédical du matériel chirurgical et des déchets $[4,6]$.

\section{Évacuation de l'instrumentation souillée et des déchets}

Avant de transporter l'instrumentation et les déchets en salle de stérilisation, ils doivent être aspergés avec un spray de solution antiseptique afin de limiter le 
développement du film bactérien et ainsi assurer une meilleure protection de l'assistante et optimiser la désinfection.

L'assistante enfile des gants épais et un masque pour effectuer un tri soigneux en séparant les instruments des déchets et les déchets mous des déchets piquants. Les déchets contaminés seront mis dans une poubelle bien séparée des déchets non contaminés (fig. 13). L'instrumentation subira toutes les étapes de la chaîne

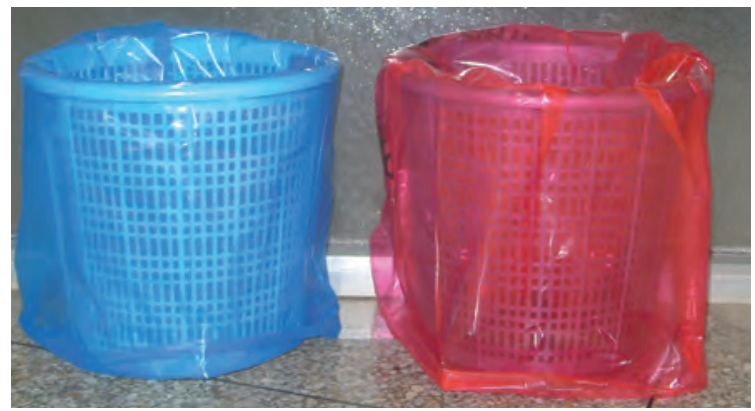

Fig. 13 :

Poubelles pour déchets. Rouge pour les déchets contaminés et bleu ou noir pour les non contaminés.

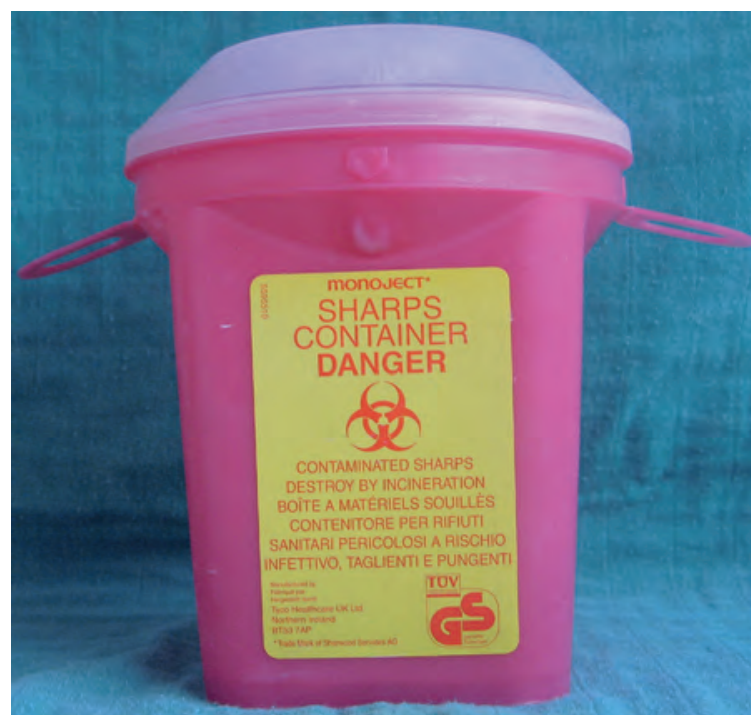

$\triangle$ Fig. 14 :

Boîte à parois rigides pour les déchets piquants ou tranchants. de stérilisation déjà mentionnées, alors que les déchets piquants seront jetés dans des containers spécifiques à parois rigides pour éviter toute piqûre contaminante $[4,6]$ (fig. 14).

\section{Entretien des locaux}

Pour réaliser un nettoyage optimal du local, il est nécessaire de retirer tous les objets de la salle d'intervention, de respecter la notion des trois zones, d'utiliser un détergent désinfectant, meilleur auteur du bionettoyage, d'insister sur la technique double seau, d'assurer une meilleure aération de la salle d'intervention $[4,6]$.

\section{Bibliographie}

[1] Martinez H, Renault P, George-Renault G, Pierrisnard L, Rouach T. Les implants : chirurgie et prothèse. Choix thérapeutique et stratégique. Éditions CdP. Wolters Kluwer France, 2008.

[2] Zeitoun R. Chirurgie implantaire : propre ou stérile ? Information dentaire, 2006.

[3] Davarpanah M, Szmukler-Moncler S, Martinez H. Manuel d'implantologie clinique : concepts, protocoles et innovations récentes. Éditions $C d P$, Wolters Kluwer France, 2008.

[4] Drouhet G, Missika P, Arnaud F. L'assistante en implantologie chirurgicale et prothétique. Éditions CdP, Wolters Kluwer France, 2008.

[5] Zeitoun R. Actualité et évolution de la pratique en stérilisation. Information dentaire 2005;87:25-29.

[6] Tazi M, Perrin D. Prévention du risque infectieux en chirurgie buccale : Asepsie-antisepsie-stérilisation. Tribune dentaire 1996;18(4): 41-47.

[7] Missika P, Drouhet G. Hygiène, asepsie, ergonomie, un défi permanent. Rueil-Malmaison : Éditions CdP, coll. JPIO, 2001.

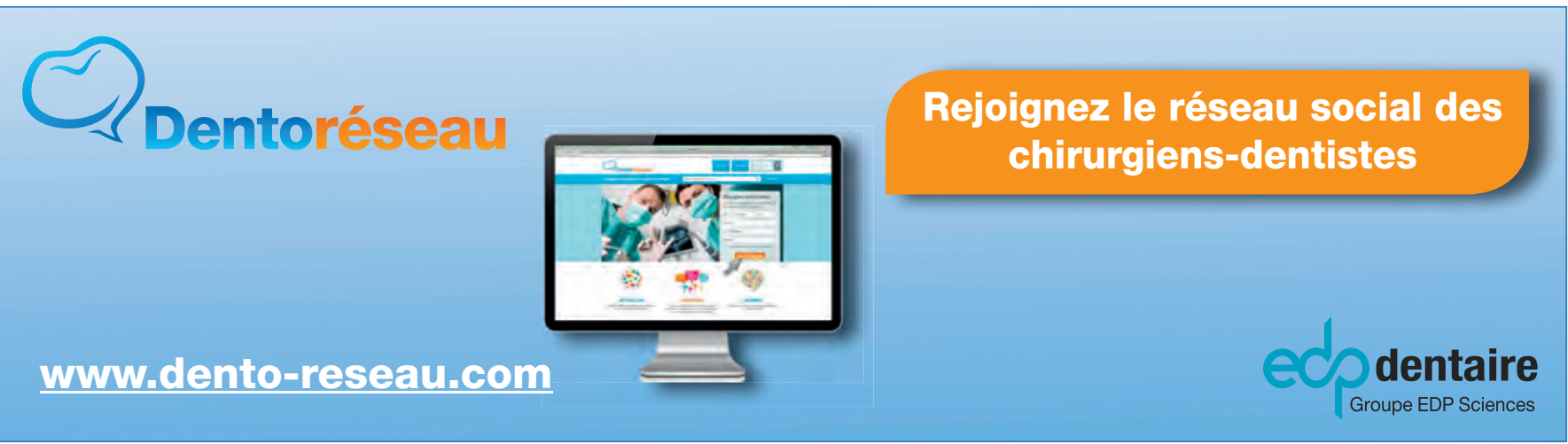

